\title{
The impact of strong recombination on temperature determination in planetary nebulae
}

\author{
V. Gomez-Llanos, ${ }^{1 \star}$ C. Morisset, ${ }^{1}$ J. García-Rojas, ${ }^{2,3}$ D. Jones, ${ }^{2,3}$ R. Wesson, ${ }^{4}$ \\ R. L. M. Corradi, ${ }^{5,2}$ and H. M.J. Boffin ${ }^{6}$ \\ ${ }^{1}$ Instituto de Astronomia (IA), Universidad Nacional Autónoma de México, Apdo. postal 106, C.P. 22800 Ensenada, Baja California, México. \\ ${ }^{2}$ Instituto de Astrofísica de Canarias, E-38200, La Laguna, Tenerife, Spain \\ ${ }^{3}$ Universidad de La Laguna. Depart. de Astrofísica, E-38206, La Laguna, Tenerife, Spain \\ ${ }^{4}$ Department of Physics and Astronomy, University College London, Gower St, London WC1E 6BT, UK \\ ${ }^{5}$ GRANTECAN, Cuesta de San José s/n, E-38712, Breña Baja, La Palma, Spain \\ ${ }^{6}$ European Southern Observatory, Karl-Schwarzschild-Str. 2, 85748 Garching bei Muenchen, Germany
}

Accepted XXX. Received YYY; in original form ZZZ

\begin{abstract}
The long-standing difference in chemical abundances determined from optical recombination lines and collisionally excited lines raises questions about our understanding of atomic physics, as well as the assumptions made when determining physical conditions and chemical abundances in astrophysical nebulae. Here, we study the recombination contribution of [O III] 4363 and the validity of the line ratio [O III] 4363/4959 as a temperature diagnostic in planetary nebulae with a high abundance discrepancy. We derive a fit for the recombination coefficient of [ $\left.\mathrm{O}_{\mathrm{III}}\right] 4363$ that takes into account the radiative and dielectronic recombinations, for electron temperatures from 200 to $30,000 \mathrm{~K}$. We estimate the recombination contribution of [O III] 4363 for the planetary nebulae Abell 46 and NGC 6778 by subtracting the collisional contribution from the total observed flux. We find that the spatial distribution for the estimated recombination contribution in [O III] 4363 follows that of the $\mathrm{O}_{\text {II }} 4649$ recombination line, both peaking in the central regions of the nebula, especially in the case of Abell 46 which has a much higher abundance discrepancy. The estimated recombination contribution reaches up to $70 \%$ and $40 \%$ of the total [O III] 4363 observed flux, for Abell 46 and NGC 6778, respectively.
\end{abstract}

Key words: atomic data, ISM: abundances - stars: AGB and post-AGB - planetary nebulae: individual: Abell 46, NGC 6778

\section{INTRODUCTION}

When measuring chemical abundances from faint heavy-element optical recombination lines (ORLs), it is found that they are always greater than those measured from the much brighter collisionally excited lines (CELs). Being known for more than 70 years, this abundance discrepancy problem is probably the most important challenge to our understanding of the physics of photoionized nebulae (see García-Rojas et al. 2019, and references therein). Several scenarios have been proposed to resolve the issue, the two most popular being: i) the existence of temperature fluctuations within a chemically homogeneous plasma (Peimbert 1967; Torres-Peimbert et al. 1980), and ii) the presence of cold, metal-rich gaseous clumps in the nebula, which are very efficiently cooled by the heavy elements (Liu et al. 2000).

However, neither of these scenarios seem appropriate to universally explain the complete range of abundance discrepancy factors (ADFs, i. e. the ratio between the abundances determined from

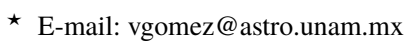

ORLs and CELs) observed in both $\mathrm{H}$ II regions and planetary nebulae (PNe; see Wesson et al. 2018) ${ }^{1}$. Moreover, the mechanisms producing, and allowing for the survival of, temperature fluctuations in a photoionized plasma are still under debate (Peimbert et al. 2017), while the same is true for the physical origin of the metal-rich component (Stasińska et al. 2007; Corradi et al. 2015). Nevertheless, some observational evidence of the existence of two or more gaseous phases in $\mathrm{PNe}$ have been found by several authors in recent years (Wesson et al. 2003; Liu et al. 2006; Wesson et al. 2008; Richer et al. 2013, 2017; Peña et al. 2017).

In particular, PNe with ADFs $>10$ have proven to be very interesting objects, as their extreme ADFs seem to be linked with the evolution of a central close-binary system that has experienced a common envelope phase (Liu et al. 2006; Corradi et al. 2015; García-Rojas et al. 2016; Jones et al. 2016; Wesson et al. 2018), even if the nature of the relationship is still a mystery. Detailed anal-

\footnotetext{
1 An updated database on the ADFs measured in $\mathrm{H}$ II regions and PNe can be found at https://www.nebulousresearch.org/adfs/
} 
ysis of the physical conditions and chemical abundances of a few objects has led several authors to suggest that the ionized gas comprises two different phases: an H-rich phase, which is dominated by Hydrogen and Helium recombination lines and collisionally excited lines (CELs) from heavy elements ( $\mathrm{O}, \mathrm{N}, \mathrm{Ne}, \mathrm{Ar} . .$.$) , alongside$ a much colder, H-poor phase with strong emission in the optical recombination lines (ORLs) of heavy elements $(\mathrm{C}, \mathrm{N}, \mathrm{O}, \mathrm{Ne})$ and almost no CEL emission (Liu et al. 2000; Wesson et al. 2005; Corradi et al. 2015; Wesson et al. 2018). Under this hypothesis, accurately determining the physical conditions (electron temperature, $T_{e}$, and electron density, $n_{e}$ ) from different CEL and ORL diagnostics is crucial to properly determine the chemical abundances in each phase. However, having two gas phase components with different chemical contents in an ionized gas complicates the computation of physical conditions and chemical abundances from an observational point of view.

A first estimate of how the presence of multiple gas components could affect the determination of physical conditions and chemical abundances in the main nebular shell was made by Liu et al. (2000), who computed new recombination coefficients for the $T_{e}$-sensitive [N $\mathrm{NI}] \lambda 5754$ and [O $\mathrm{II}] \lambda \lambda 7320+30$ auroral lines and found that recombination excitation was important in exciting these lines and that ignoring it would lead to an overestimated $T_{e}$. These authors also proposed a fit to the contribution of radiative recombination to the widely used [O III] $\lambda 4363$ auroral line, valid for $T_{e}>8,000 \mathrm{~K}$.

In this letter we want to explore the classical [O $\mathrm{III}]$ $\lambda 4363 / \lambda 4959 T_{e}$ diagnostic, that can be strongly contaminated by recombination in extreme $\mathrm{ADF} \mathrm{PNe}$ and, therefore, is no longer suitable for the measurement of $T_{e}$. In this work we try to determine the contribution of the recombination to the [O III] $\lambda 4363$ line, for the PNe NGC 6778 and Abell 46. In $\S 2$ we briefly describe the observational data used in this paper; in $\S 3$ we present new calculations to compute the recombination contribution to the [O III] $\lambda 4363$ CEL emissivity; in $\S 4$ we estimate the recombination contribution from an observational point of view and, finally, in $\S 5$ we discuss our results.

\section{OBSERVATIONS}

We have used long-slit, intermediate-resolution spectra taken by our group of the extreme ADF planetary nebulae NGC 6778 (with FORS2-VLT 8.2m; see Jones et al. 2016, ADF 18) and Abell 46 (with ISIS-WHT 4.2m; see Corradi et al. 2015, ADF 120) respectively. The FORS2 observations covered the wavelength range $3600-5000 \AA$ with an average spectral resolution of $1.5 \AA$. The ISIS observations covered the wavelength range 3610-5050 $\AA$ with a spectral resolution of $0.8 \AA$. For additional details on the observations and data reduction, we refer the reader to the original references.

For each long slit, we split the 2D-spectrum into several spatial bins along the slit $-2^{\prime \prime} .5$ and $0^{\prime \prime} .5$ wide for Abell 46 and NGC 6778 , respectively- which provides enough signal to noise for the faintest lines of interest to be measured. The fluxes of the [O III] $\lambda 4363$ and $\lambda 4959$ CELs and the O II $\lambda \lambda 4649+50$ ORL are obtained by automatically fitting Gaussian profiles to each line (for the ORL line, a double Gaussian is used to take into account the two members of the multiplet at $4649.13 \AA$ and $4650.25 \AA$; we ignore the contribution of C III $\lambda 4650.25$ because other lines of the same multiplet such as C III $\lambda 4647.42$ have not been reported in the literature spectra of either object (Jones et al. 2016; Corradi et al. 2015). The uncertain- ties are determined in each spatial bin through a quadratic mean of the difference between the Gaussian fit and the signal.

In Fig. 1, we show examples of the line fitting process for the $2 \mathrm{PNe}$ considered in this paper (upper panels for Abell 46 and lower panels for NGC 6778).

\section{THE LIMITS IN COMPUTING [O III] $\lambda 4363$ INTENSITY}

To compute the [O III] $\lambda 4363$ emission, we first consider the contribution from the radiative deexcitation of the $\mathrm{O}^{++}$ion following an excitation of the level ${ }^{1} \mathrm{~S}_{0}$ by collision with a free electron of the plasma. This is obtained using PyNeb (Luridiana et al. 2015) version 1.1.10, based on collision strengths by Storey et al. (2014) and transition probabilities by Froese Fischer \& Tachiev (2004).

We also have to carefully take into account the radiative recombination (RR) computed by the fit from Pequignot et al. (1991) and the dielectronic recombination (DR) from Nussbaumer \& Storey (1984).

A fit for the total recombination contribution is given by Liu et al. (2000) as:

$\frac{I(4363)}{I(H \beta)}=12.4 \times t^{0.59} \times \frac{O^{3+}}{H^{+}}$.

This line ratio $[\mathrm{O}$ III] $\lambda 4363 / \mathrm{H} \beta$ leads to a recombination coefficient of the single [O III] $\lambda 4363$ line close to:

$\alpha_{4363}\left[\mathrm{~cm}^{3} \mathrm{~s}^{-1}\right] \simeq 3.3 \times 10^{-13} \times t^{-0.21}$,

where $\mathrm{t}=\mathrm{T}_{e} / 10^{4} \mathrm{~K}$ and considering $\alpha_{\mathrm{H} \beta} \simeq 2.94 \times 10^{-14} \times$ $t^{-0.80} \mathrm{~cm}^{3} \mathrm{~s}^{-1}$.

This fit has been obtained when the DR has a considerable effect on the total recombination. This occurs for $T_{e}$ between $8,000 \mathrm{~K}$ and $20,000 \mathrm{~K}$. For $T_{e}<5,000 \mathrm{~K}$, the DR is negligible compared to the RR and the dependency on $T_{e}$ does not follow the fit by Liu et al. (2000) anymore. We computed a new fit that reproduces the sum RR+DR within $3 \%$ from 200 to $30,000 \mathrm{~K}$ :

$\alpha_{4363} \simeq 2.63 \times 10^{-13} \times t^{-0.6}+1.4 \times 10^{-13} \times e^{-0.8 / t}$.

This fit is used in the multipurpose photoionization code Cloudy (Ferland et al. 2017) since v17.02. In Fig. 2, we can see the variation of recombination coefficients with $T_{e}$ for the RR and DR computed from Pequignot et al. (1991) and Nussbaumer \& Storey (1984) respectively, as well as the fit by Liu et al. (2000) and our fit from Eq. 3. Note that the recombination computed in Cloudy until v17.02 is rather overestimated as it uses Burgess \& Seaton (1960) upper limits (purple line in Fig. 2).

It is important to notice here that in general, no simple fit to the line ratio $[\mathrm{O}$ III] $\lambda 4363 / \mathrm{H} \beta$ (like the one obtained by Liu et al. (2000), Eq. 1 above) can be obtained, as the regions where the recombination lines [O III] $\lambda 4363$ and $\mathrm{H} \beta$ are produced can be very different, especially in terms of temperature, densities and volume. The complete relation is :

$\frac{I(4363)}{I(\beta)}=\frac{\int_{V} E_{4363} \alpha_{4363}(T) n\left(O^{3+}\right) n(e) d V}{\int_{V} E_{\beta} \alpha_{\beta}(T) n\left(H^{+}\right) n(e) d V}$,

where $E_{\lambda}$ is the energy of the corresponding emission line and $n(X)$ is the density (by number) of the ion $X$ responsible for the line emission (namely $\mathrm{O}^{3+}$ and $\mathrm{H}^{+}$in the present case). 

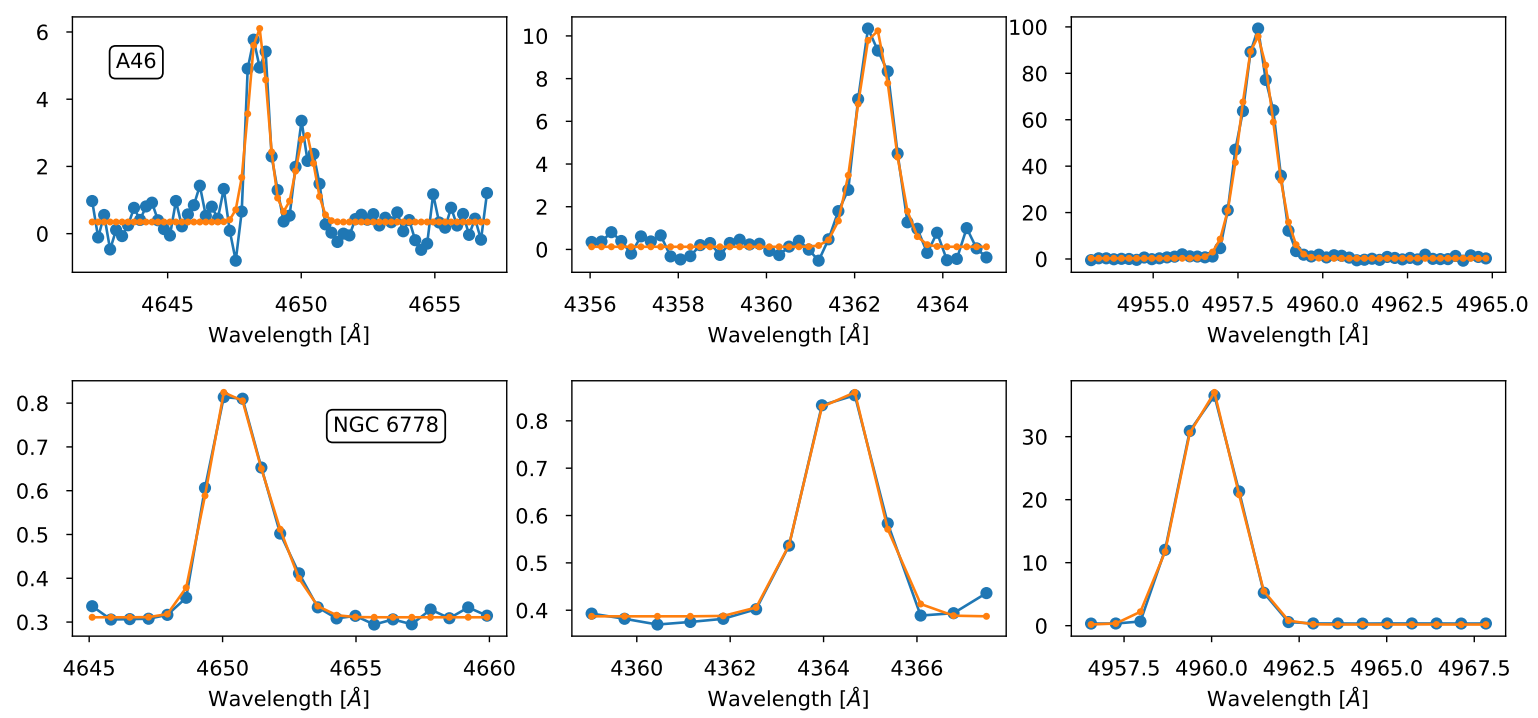

Figure 1. Examples of the fit to the emission lines. Upper panels for A46 and lower panels for NGC 6778 . From left to right, the fits are for the O II $\lambda \lambda 4649+50$, the $[\mathrm{O}$ III] $\lambda 4363$ and the $[\mathrm{O} \mathrm{III}] \lambda 4959$ emission lines. Line fluxes are in arbitrary units.

\section{TESTING BI-METALLICITY HYPOTHESIS}

In the following we explore the case where the nebula is made of two regions of very different abundances. Then, the $\mathrm{H} \beta$ line is mainly emitted by the close-to-solar metallicity region 1 and the [O III] $\lambda 4363$ recombination line is mainly emitted by a cold, metal-rich region 2, equation 4 leads to:

$$
\frac{I(4363)}{I(\beta)}=\frac{E_{4363} \alpha_{4363}\left(T_{2}\right) n\left(O^{3+}\right)_{2} n(e)_{2} V_{2}}{E_{\beta} \alpha_{\beta}\left(T_{1}\right) n\left(H^{+}\right)_{1} n(e)_{1} V_{1}},
$$

where the 1 and 2 subscripts indicate a mean value over the regions 1 and 2 respectively ${ }^{2}$.

Therefore, if $T_{1}$ and $T_{2}$ are very different, the simplification of the temperature dependent power terms in the recombination coefficients of the two lines cannot be applied, nor do the $n_{e}$ ratio $n(e)_{1} / n(e)_{2}$ and the volume ratio $V_{1} / V_{2}$ cancel. These ratios cancel only if the same region of the nebula is considered to emit both lines. The abundance ratio $\mathrm{O}^{3+} / \mathrm{H}^{+}$only appears in a final relation if the implicit hydrogen densities $n(H)_{1}$ and $n(H)_{2}$ are the same.

Gómez-Llanos \& Morisset (2020) explored a case where an $\operatorname{ADF}\left(\mathrm{O}^{2+}\right) \sim 8$, determined from observations of NGC 6153, can be reproduced by models in which the actual abundance ratio between the two components (termed the abundance contrast factor or ACF) is as high as 600. In their Annex, they even show that an ACF of 1,000 could lead to an apparent ADF of 1 !

As derived from Eqs. 5 and 6 above, it is very difficult to determine the contribution to the emission of the [O III] $\lambda 4363$ that comes from the recombination in cases where the gas has two phases of different metallicities, with the metal recombination contribution

2 In the general situation, both lines are emitted by both regions and the line intensities are obtained by summing contributions from region 1 and 2, leading to an even more complex equation for the line ratio:

$\frac{I(4363)}{I(\beta)}=\frac{E_{4363}\left[I(4363)_{1}+I(4363)_{2}\right]}{E_{\beta}\left[I(\beta)_{1}+I(\beta)_{2}\right]}$,

where $I(\lambda)_{i}=\alpha_{\lambda}\left(T_{i}\right) n(X)_{i} n(e)_{i} V_{i}$.

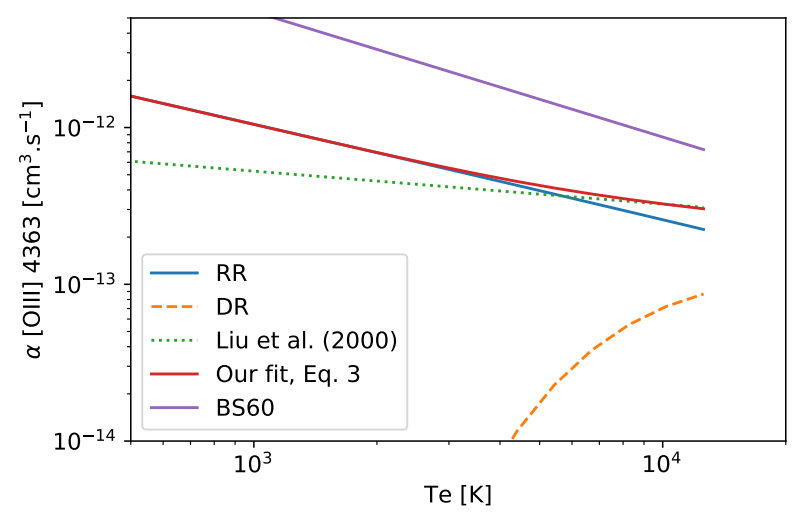

Figure 2. Recombination coefficients of [O III] 14363 : radiative recombination (RR) computed by Pequignot et al. (1991) (blue line), dielectronic recombination (DR) by Nussbaumer \& Storey (1984) (orange dashed line), the value obtained by the formula from Liu et al. (2000) (Eq. 1, green dot line) and our fit to RR+DR (Eq. 3, red dot-dashed line). The actual value of RR+DR is not shown, as it is not distinguishable from our fit. The BS60 values from Burgess \& Seaton (1960) upper limits used in Cloudy are also shown in purple.

mainly coming from the H-poor region. Estimating the parameters (e.g. $T_{e}$ and $n_{e}$ ) of both regions needed in Eq. 5 is very hard, as for most objects the observed morphology does not allow to separate the emission coming from each region.

We have seen that, from a theoretical stand-point, it is almost impossible to correctly determine the contribution to the emission of the [O III] $\lambda 4363$ line that originates from recombination. We can nevertheless attempt to obtain this contribution on an observational basis. In the following, we try to determine the recombination contribution by removing the contribution of the collisionally excited emission to the total emission.

Several authors have found that the spatial distribution of the [O III] $\lambda 4363$ emission is very similar to that of the $\mathrm{O}_{\text {II }} \lambda 4649$ 
one, but very different from the [O III] $\lambda 5007,4959$ lines (Corradi et al. 2015; García-Rojas et al. 2016; Jones et al. 2016; Wesson et al. 2018). The observed behavior is consistent with an increasing temperature towards the central parts of the PN, which is at odds with the fact that $\mathrm{O}_{\text {II }}$ ORL emission also peaks at the center of the nebula, indicating that the cold, H-poor gas is located close to the central star (García-Rojas et al. 2016).

Adopting a $n_{e}$ of $10^{3} \mathrm{~cm}^{-3}$, we estimate (using PyNeb, version 1.1.10) the spatial distribution of $T_{e}$ in Abell 46 and NGC 6778 from the line ratio [O $\mathrm{III}] \lambda 4363 / 4959$. This is shown in blue in the top left and right panels of Fig. 4, respectively. In Abell 46, we can see a noticeable increase of the temperature estimation towards the center of the object. In the left and middle panels of Fig. 3, we show MUSE emission line maps of the $\mathrm{T}_{e}$ sensitive [S III] $\lambda 6312$ and [S III] $\lambda 9068$ CELs in NGC 6778 (García-Rojas et al. in prep.), and in the right panel, we show the $T_{e}$ map obtained from the ratio of both lines, assuming a constant density $n_{e} \sim 1,000 \mathrm{~cm}^{-3}$. The $T_{e}$ map shows a roughly constant $T_{e}$ distribution, with an average $T_{e}$, weighted by the [S $\mathrm{SII}] \lambda 6312$ flux of $\sim 8,150 \mathrm{~K}$, implying that $\mathrm{S}^{3+}$ recombination emission is not significantly enhanced. This is consistent with previous studies, which suggested that the phenomenon of highly enhanced recombination-line emission is restricted to second-row elements (Barlow et al. 2003; Wesson et al. 2018). Following this observational evidence and the bi-metallicity hypothesis, we adopt a constant $T_{e}$ (red solid line in top panels of Fig. 4) for the close-tosolar region in Abell 46 and NGC 6778 of 10,000 K and 8,000 K (see above), respectively. The spatial distribution of the observed [O III] $\lambda 4363$ line, is plotted in orange in the middle panels of Fig. 4 for Abell 46 (left) and NGC 6778 (right). To obtain the collisional contribution of [ $\left.\mathrm{O}_{\mathrm{III}}\right] \lambda 4363$, we divide the observed emission of the line [O III] $\lambda 4959$ by the theoretical line ratio [O III] $\lambda \lambda 4959 / 4363$ at the adopted constant temperature ${ }^{3}$. The result is shown in green in the middle panels of Fig. 4. We then subtract this collisional contribution to the total observed flux of [O III] $\lambda 4363$ (orange line) to get the possible recombination contribution of $\lambda 4363{\text { from } \mathrm{O}^{+3}}^{+3}$ (red line). For comparison, we also show the spatial distribution of O II $\lambda 4649$ ORL (blue line) multiplied by a normalization factor. We can see that the residual spatial profile of [O III] $\lambda 4363$ (red line) resembles that of the $\mathrm{O}$ II $\lambda 4649 \mathrm{ORL}$, indicating that the emissivity of the line is dominated by the recombination contribution. In the lower panels of Fig. 4, we show the spatial distribution of the recombination contribution to the total [O III] $\lambda 4363$, which reaches up to $70 \%$ and $50 \%$ of the total emission for Abell 46 (left) and NGC 6778 (right), respectively.

The recombination contribution to the [O III] $\lambda 4363$ line may also be estimated using $\mathrm{O}^{3+}$ ORLs. Using Pequignot et al. (1991) via PyNeb, one can for example deduce I([O III] $\lambda 4363) / \mathrm{I}(\mathrm{O}$ III $\lambda 3762+)$ increasing from 0.35 to 0.55 ( 0.45 to 0.7 ) when $T_{e}$ increases from $1,000 \mathrm{~K}$ to $20,000 \mathrm{~K}$ in case B (case A resp.). Jones et al. (2016) report $\mathrm{I}(\mathrm{O}$ III $\lambda 3760)=0.61(\mathrm{H} \beta=100)$ for NGC 6778. One can estimate the intensity of the whole $\mathrm{V} 2$ multiplet $\mathrm{I}(\mathrm{O}$ III $\lambda 3762+)$ to be $\simeq 1.00$, leading to a prediction of $\mathrm{I}([\mathrm{O} \mathrm{III}]$ 14363) from recombination to be of the order of 0.4 to 0.5 . This is between 20 to $25 \%$ of the observed $\mathrm{I}([\mathrm{O}$ III $] 24363)=2.07$, close to what we obtain for the same PN (see Fig. 4).

\footnotetext{
3 This is obtained with PyNeb, from the ratio of emissivities $\varepsilon(4959) / \varepsilon(4363)$ using the adopted temperature of $8,000 \mathrm{~K}$ and $10,000 \mathrm{~K}$ for NGC 6778 and Abell 46 respectively, and a density of $10^{3} \mathrm{~cm}^{-3}$. The corresponding ratios are 117 and 51 .
}

\section{DISCUSSION}

In this paper we explored the very crude hypothesis that the $T_{e}$ of the close-to-solar abundance gas in the central part of the nebula is the same as in the main nebula (the red line showing the adopted value in Fig. 4). This may not be the case. If one wants to increase the precision in the determination of the [O $\mathrm{III}] \lambda 4363$ recombination contribution, one needs to make a detailed photoionization model of the object. This requires a good atmosphere model for the ionizing source in order to correctly reproduce the heating of the nebula. One also may need to take into account the presence of dust and its properties, to accurately compute the balance between the heating and the cooling in the inner part of the nebula. This is totally out of the scope of the simple "proof of concept" presented in this letter.

One can also question the precision of the atomic data involved in the different parts of the emission calculus, especially the radiative recombination at low temperature. Although the dielectronic recombination seems to vanish at low temperature (Fig. 2), it has recently been pointed out that the effect of "exotic" atomic processes like Rydberg Enhanced Recombination (RER) could be very important in these regimes. RER could thus have an impact on the predicted ionization balance and, hence, on the derived ionic abundances (Nemer et al. 2019), changing $n\left(O^{3+}\right)$ in Eq. 5. The residual obtained in Sec. 4 and associated to the $O^{+}$recombination can also include a contribution from RER, as such an exact computation of the RER-based emission will be important in understanding the entirety of the [O III] $\lambda 4363$ emission.

Regarding observations, it is becoming increasingly clear that for a complete understanding of this problem, a combination of detailed photoionization models with deep IFU observations might improve the situation. In the case of extreme ADF PNe, were two different plasma components coexist, the Balmer and Paschen jumps might not be indicative of any real gas temperature, as they are only a weighted mean of two very different phases of gas. Similarly, the recombination lines (e.g. $\mathrm{O}_{\text {II }}$ or $\mathrm{H}_{\mathrm{I}}$ ) are not telling us the value of the ionic abundance ratio $\mathrm{O}^{2+} / \mathrm{H}^{+}$, as it is impossible to determine what fraction of $\mathrm{H}^{+}$actually comes from the cold region. The exact weight of the H-poor zone can only be constrained through detailed photoionization models. From the comparison of theoretical models with observations, one can obtain the physical properties $\left(T_{e}, n_{e}\right.$, mass, abundances) of the two plasma components that reproduce the observed spectra. However, a detailed treatment of the physics has revealed that the ADF might be only a rough estimate of this discrepancy and is unlikely to provide "real" information on the ORL/CEL abundance ratios (see Gómez-Llanos \& Morisset 2020).

\section{ACKNOWLEDGMENTS}

The authors thank the referee for their comments. This paper is based on observations made with ESO Telescopes at the Paranal Observatory under programme IDs 093.D-0038 and 097.D-0241, and on observations made with the William Herschel Telescope operated on the island of La Palma by the Isaac Newton Group of Telescopes in the Spanish Observatorio del Roque de los Muchachos of the Instituto de Astrofísica de Canarias. VG-L, CM acknowledges support from projects CONACyT / CB2015 - 254132 and UNAM / PAPIIT - IN101220. JG-R acknowledges support from an Advanced Fellowship from the Severo Ochoa excellence program (SEV-2015-0548). JG-R, DJ and RC acknowledge support from the State Research Agency (AEI) of the Spanish Ministry of Science, Innovation and Universities (MCIU) and the European Regional Development Fund (FEDER) under grant AYA2017-83383-P. 

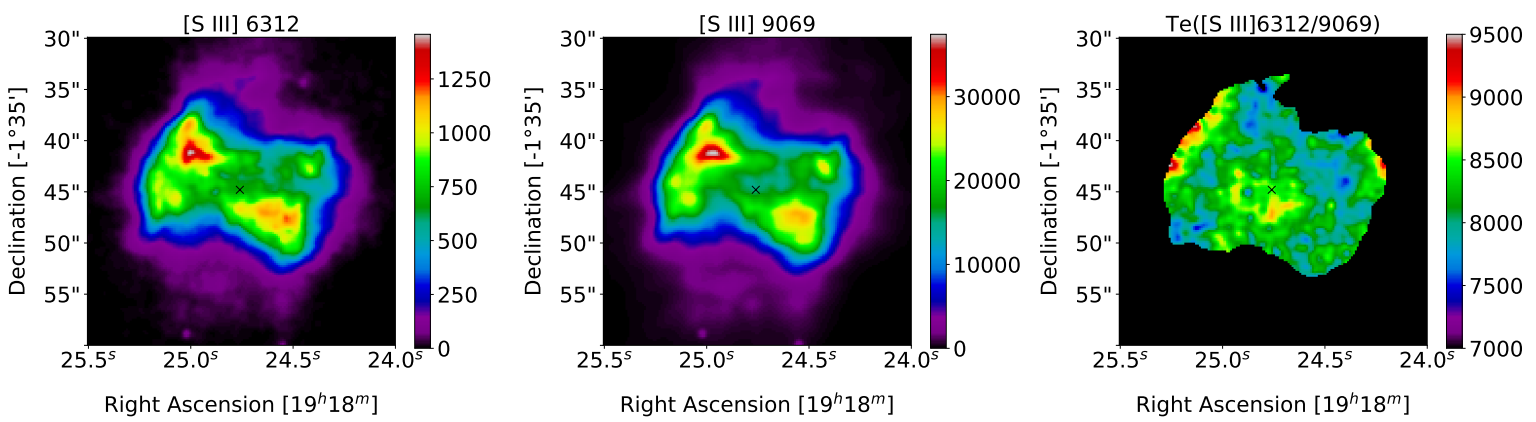

Figure 3. Left and middle panels: MUSE emission line derredened maps of the $T_{e}$ sensitive [S III] $\lambda 6312$ and [S III] $\lambda 9068$ CELs for NGC 6778 , showing a very similar spatial distribution. The raw maps have been convolved with a gaussian kernel with $\sigma=1.5$ pixels. The " $x$ " marks the position of the central star in both maps. The colorbar shows the flux in units of $10^{-20} \mathrm{erg} \mathrm{s}^{-1} \mathrm{~cm}^{-2} \AA^{-1}$. Right panel: $T_{e}$ ([S III]) map computed with PyNeb from the extinction corrected [S III] $\lambda 6312 / \lambda 9068$ line ratio (a cut is made for intensities lower than $10 \%$ of the peak emission in [S III] $\lambda 6312$ ). The average $T_{e}$ weighted by the flux of the $\lambda 6312$ line is $\sim 8150 \mathrm{~K}$.
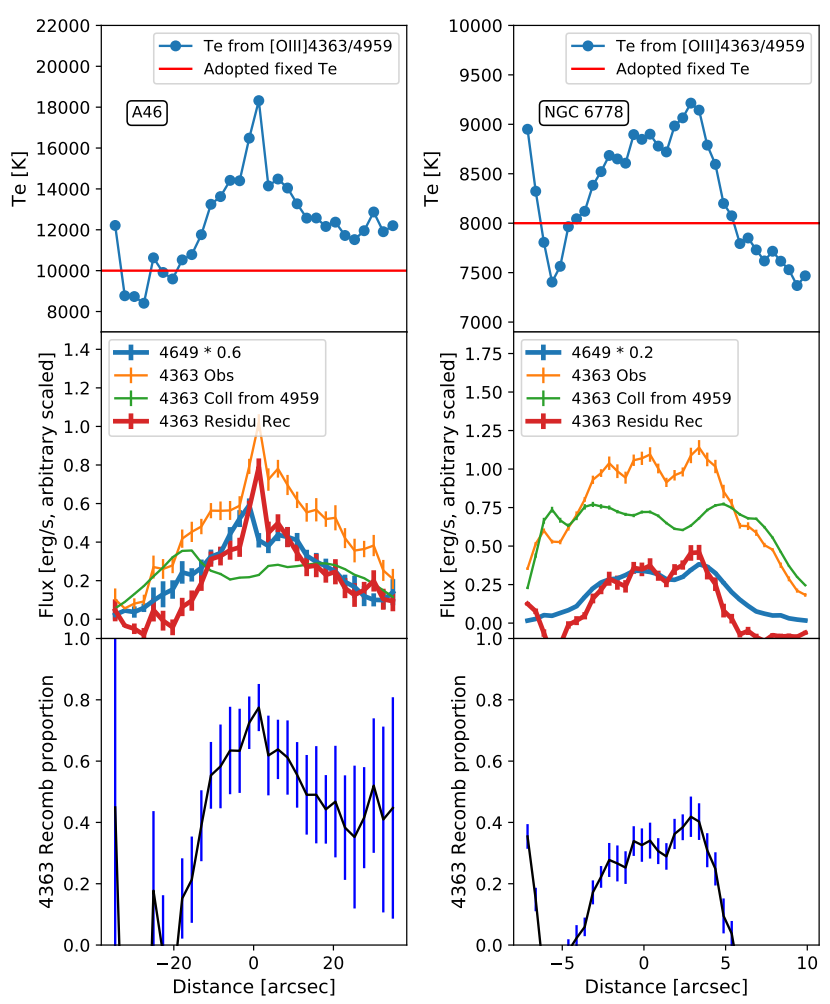

Figure 4. Top panels: $T_{e}$ estimated from [O III] $\lambda 4363 / \lambda 4959$ ratio. The red line represents the adopted $T_{e}$. Middle panels: Spatial distribution of observed [O III] $\lambda 4363$ (orange), the expected profile of [O III] $\lambda 4363$ emitted by collision assuming the fixed $T_{e}$ from the upper panels and the profile of [O III] $\lambda 4959$ (green), the residuals from subtracting the expected collisional [O III] $\lambda 4363$ profiles from the observed one (red), and for comparison the $\mathrm{O}$ II $\lambda 4649$ profile multiplied by a scale factor (blue). Lower panels: Relative contribution of recombination to the [O III] $\lambda 4363$ line.

\section{DATA AVAILABILITY}

The original data used in the paper are available under request to the authors.

\section{REFERENCES}

Barlow M. J., Liu X. W., . Péquignot D., Storey P. J., Tsamis Y. G., Morisset C., 2003, in Kwok S., Dopita M., Sutherland R., eds, IAU Symposium Vol. 209, Planetary Nebulae: Their Evolution and Role in the Universe. p. 373

Burgess A., Seaton M. J., 1960, MNRAS, 120, 121

Corradi R. L. M., García-Rojas J., Jones D., Rodríguez-Gil P., 2015, ApJ, 803,99

Ferland G. J., et al., 2017, Rev. Mex. Astron. Astrofis., 53, 385

Froese Fischer C., Tachiev G., 2004, Atomic Data and Nuclear Data Tables, 87,1

García-Rojas J., Corradi R. L. M., Monteiro H., Jones D., Rodríguez-Gil P., Cabrera-Lavers A., 2016, ApJ, 824, L27

García-Rojas J., Wesson R., Boffin H. M. J., Jones D., Corradi R. L. M., Esteban C., Rodríguez-Gil P., 2019, AAA Workshop Series, 11, 33

Gómez-Llanos V., Morisset C., 2020, MNRAS, submitted

Jones D., Wesson R., García-Rojas J., Corradi R. L. M., Boffin H. M. J., 2016, MNRAS, 455, 3263

Liu X.-W., Storey P. J., Barlow M. J., Danziger I. J., Cohen M., Bryce M., 2000, MNRAS, 312, 585

Liu X. W., Barlow M. J., Zhang Y., Bastin R. J., Storey P. J., 2006, MNRAS, 368, 1959

Luridiana V., Morisset C., Shaw R. A., 2015, A\&A, 573, A42

Nemer A., et al., 2019, ApJ, 887, L9

Nussbaumer H., Storey P. J., 1984, A\&AS, 56, 293

Peña M., Ruiz-Escobedo F., Rechy-García J. S., García-Rojas J., 2017, MNRAS, 472,1182

Peimbert M., 1967, ApJ, 150, 825

Peimbert M., Peimbert A., Delgado-Inglada G., 2017, PASP, 129, 082001

Pequignot D., Petitjean P., Boisson C., 1991, A\&A, 251, 680

Richer M. G., Georgiev L., Arrieta A., Torres-Peimbert S., 2013, ApJ, 773, 133

Richer M. G., Suárez G., López J. A., García Díaz M. T., 2017, AJ, 153, 140

Stasińska G., Tenorio-Tagle G., Rodríguez M., Henney W. J., 2007, A\&A, 471,193

Storey P. J., Sochi T., Badnell N. R., 2014, MNRAS, 441, 3028

Torres-Peimbert S., Peimbert M., Daltabuit E., 1980, ApJ, 238, 133

Wesson R., Liu X. W., Barlow M. J., 2003, MNRAS, 340, 253

Wesson R., Liu X. W., Barlow M. J., 2005, MNRAS, 362, 424

Wesson R., Barlow M. J., Liu X. W., Storey P. J., Ercolano B., De Marco O., 2008, MNRAS, 383, 1639

Wesson R., Jones D., García-Rojas J., Boffin H. M. J., Corradi R. L. M., 2018, MNRAS, 480, 4589

This paper has been typeset from a $\mathrm{TE}_{\mathrm{E}} \mathrm{X} / \mathrm{L} \mathrm{T} \mathrm{X}$ file prepared by the author. 